Cite this: Phys. Chem. Chem. Phys., 2014, 16, 3122

Received 18th October 2013, Accepted 23rd December 2013

DOI: $10.1039 / c 3 c p 54418 d$

www.rsc.org/pccp

\title{
New insight into the potential energy landscape and relaxation pathways of photoexcited aniline from CASSCF and XMCQDPT2 electronic structure calculations $\dagger$
}

\author{
Matthieu Sala, ${ }^{a}$ Oliver M. Kirkby, ${ }^{b}$ Stéphane Guérin ${ }^{a}$ and Helen H. Fielding*b
}

\begin{abstract}
There have been a number of recent experimental investigations of the nonadiabatic relaxation dynamics of aniline following excitation to the first three singlet excited states, $1^{1} \pi \pi^{\star}, 1^{1} \pi 3 \mathrm{~s} / \pi \sigma^{\star}$ and $2^{1} \pi \pi^{*}$. Motivated by differences between the interpretations of experimental observations, we have employed CASSCF and XMCQDPT2 calculations to explore the potential energy landscape and relaxation pathways of photoexcited aniline. We find a new prefulvene-like $\mathrm{MECl}$ connecting the $1^{1} \pi \pi^{*}$ state with the GS in which the carbon-atom carrying the amino group is distorted out-of-plane. This suggests that excitation above the $1^{1} \pi 3 s / \pi \sigma^{*}$ vertical excitation energy could be followed by electronic relaxation from the $1^{1} \pi \pi^{*}$ state to the ground-electronic state through this $\mathrm{MECl}$. We find a $\mathrm{MECl}$ connecting the $1^{1} \pi 3 \mathrm{~s} / \pi \sigma^{*}$ and $1^{1} \pi \pi^{*}$ states close to the local minimum on $1^{1} \pi 3 \mathrm{~s} / \pi \sigma^{*}$ which suggests that photoexcitation to the $1^{1} \pi 3 s / \pi \sigma^{*}$ state could be followed by relaxation to the $1^{1} \pi \pi^{\star}$ state and to the dissociative component of the $1^{1} \pi 3 s / \pi \sigma^{*}$ state. We also find evidence for a new pathway from the $2^{1} \pi \pi^{*}$ state to the ground electronic state that is likely to pass through a three-state conical intersection involving the $2^{1} \pi \pi^{\star}, 1^{1} \pi 3 \mathrm{~s} / \pi \sigma^{\star}$ and $1^{1} \pi \pi^{\star}$ states.
\end{abstract}

\section{Introduction}

Aniline is the simplest aromatic amine and, like all aromatic molecules containing $\mathrm{OH}$ and $\mathrm{NH}$ groups, it has a remarkably low fluorescence quantum yield attributed to highly efficient radiationless electronic relaxation pathways connecting the excited electronic states to the ground electronic state. Improving our understanding of the mechanism of electronic relaxation in small molecules like aniline is an important component of the toolkit required to design photostable materials from first principles.

Aniline absorbs UV radiation around $282 \mathrm{~nm}(4.4 \mathrm{eV})$ and $230 \mathrm{~nm}(5.4 \mathrm{eV}) \mathrm{nm} .{ }^{1-3}$ These bands arise from transitions from the ground electronic state to the two lowest lying ${ }^{1} \pi \pi^{*}$ states.

\footnotetext{
${ }^{a}$ Laboratoire Interdisciplinaire Carnot de Bourgogne UMR 5209 CNRS, Université de Bourgogne, BP 47870, F-21078 Dijon, France.

E-mail: matthieu.sala@u-bourgogne.fr

${ }^{b}$ Department of Chemistry, University College London, 20 Gordon Street, London WC1H OAJ, UK. E-mail: h.h.fielding@ucl.ac.uk

$\dagger$ Electronic supplementary information (ESI) available: Containing figures showing the orbitals, the negative imaginary frequency normal modes for the transition states and the branching plane vectors for the minimum energy conical intersections. The Cartesian coordinates and selected geometrical parameters for all the stationary points optimized in this work are also reported. See DOI: $10.1039 / \mathrm{c} 3 \operatorname{cp} 54418 \mathrm{~d}$
}

There have been numerous experimental ${ }^{4-7}$ and theoretical ${ }^{8-22}$ studies of the ground electronic state, with particular focus on the pyramidal geometry of the amino group. There has been some controversy over the equilibrium geometry of the $1^{1} \pi \pi^{*}$ excited state. Configuration-interaction with single excitations (CIS) calculations predicted planar or pyramidal geometries with a smaller degree of pyramidalization than the ground state, depending on basis set. ${ }^{23-31}$ CASSCF calculations also predicted a pyramidal equilibrium geometry. ${ }^{32}$ Rotationally-resolved electronic spectra suggested a quasiplanar geometry for the $1^{1} \pi \pi^{*}$ excited state; however, this structure was a vibrationally averaged structure rather than the true minimum of the potential energy surface (PES). ${ }^{30}$ Early experimental investigations of the photochemistry and photophysics of aniline following excitation of the $1^{1} \pi \pi^{*}$ excited state focussed on fluorescence and intersystem crossing (ISC). ${ }^{33-36}$

Between the first two ${ }^{1} \pi \pi^{*}$ states, lies a ${ }^{1} \pi 3 \mathrm{~s} / \pi \sigma^{*}$ state that has significant Rydberg character in the Franck-Condon (FC) region and evolves into a valence state with dissociative character along the $\mathrm{N}-\mathrm{H}$ stretching coordinate. ${ }^{37-39}$ Worth et al. have predicted that two $3 \mathrm{p}$ Rydberg states also lie between the two ${ }^{1} \pi \pi^{*}$ states. ${ }^{40}$ There have been a number of experimental ${ }^{32,41-45}$ and theoretical ${ }^{32,40}$ investigations focussing on electronic relaxation pathways involving the $1^{1} \pi 3 \mathrm{~s} / \pi \sigma^{*}$ state. Experiments observing the formation of $\mathrm{H}$-atoms following photodissociation 
reported thresholds for fast $\mathrm{H}$-atom production (a signature of $\mathrm{NH}$ bond fission on the $1^{1} \pi \sigma^{*}$ surface) of $260 \mathrm{~nm}^{41}$ and $250 \mathrm{~nm}^{32}$ depending on whether they employed nanosecond or femtosecond lasers, respectively. Femtosecond pump-probe experiments have revealed four lifetimes: $\tau_{1} \lesssim 100 \mathrm{fs}, \tau_{2} \sim 100-400 \mathrm{fs}, \tau_{3} \sim 0.4-3 \mathrm{ps}$, $\tau_{4} \gtrsim 80 \mathrm{ps}^{32,42-45}$ The fastest timescale, $\tau_{1}$, is only observed following excitation to the $2^{1} \pi \pi^{*}$ excited state and has been interpreted as relaxation back to the electronic ground-state ${ }^{43,44}$ or as decay through a series of conical intersections (CIs) to the $1^{1} \pi \sigma^{*}$ PES. $^{32,42} \tau_{2}$ has been interpreted as population transfer through a CI between the $1^{1} \pi 3 \mathrm{~s} / \pi \sigma^{*}$ state and the $1^{1} \pi \pi^{*}$ state governing a competition between subsequent relaxation on the $1^{1} \pi \pi^{*}$ state and the dissociative $1^{1} \pi \sigma^{*}$ component of the $1^{1} \pi 3 \mathrm{~s} / \pi \sigma^{*}$ state, ${ }^{43,44}$ or the reverse process from $1^{1} \pi \pi^{*}$ to $1^{1} \pi \sigma^{*}{ }^{32,42} \tau_{2}$ has also been proposed to arise from tunnelling through the barrier along the $\mathrm{N}-\mathrm{H}$ stretching coordinate between the bound $1^{1} \pi 3 \mathrm{~s}$ and dissociative $1^{1} \pi \sigma^{*}$ components of the $1^{1} \pi 3 s / \pi \sigma^{*}$ state $^{45} \tau_{3}$ was observed only in time-resolved photoelectron spectroscopy experiments ${ }^{43-45}$ and was interpreted as motion on the $1^{1} \pi 3 \mathrm{~s} / \pi \sigma^{*}$ $\mathrm{PES}^{43,44}$ or as intramolecular vibrational energy redistribution (IVR) in the $1^{1} \pi \pi^{*}$ state. ${ }^{45}$ The longest timescale, $\tau_{4}$, has been interpreted as decay from the $1^{1} \pi \pi^{*}$ state.

Motivated by the disagreements between the interpretations of these experiments, we have employed CASSCF and XMCQPDT2 calculations to explore the potential energy landscape and relaxation pathways of aniline following photoexcitation to the $1^{1} \pi \pi^{*}$, $1^{1} \pi 3 \mathrm{~s} / \pi \sigma^{*}$ and $2^{1} \pi \pi^{*}$ states. We find a new prefulvene-like minimum energy conical intersection (MECI) connecting the $1^{1} \pi \pi^{*}$ state with the GS which seems likely to be involved in nonradiative decay from $1^{1} \pi \pi^{*}$ to the ground-state. We find a MECI connecting the $1^{1} \pi 3 \mathrm{~s} / \pi \sigma^{*}$ and $1^{1} \pi \pi^{*}$ states close to the local minimum on $1^{1} \pi 3 \mathrm{~s} / \pi \sigma^{*}$ which suggests that excitation to $1^{1} \pi 3 \mathrm{~s} /$ $\pi \sigma^{*}$ is likely to be followed by relaxation to this MECI where population will subsequently be transferred both to $1^{1} \pi \pi^{*}$ (as observed in our earlier work ${ }^{43,44}$ ) and to the dissociative component of $1^{1} \pi 3 \mathrm{~s} / \pi \sigma^{*}$ (as observed by Stavros et al. ${ }^{32}$ ). Finally, we find evidence for a new decay pathway connecting $2^{1} \pi \pi^{*}$ and the ground-state that passes through what seems likely to be a three-state CI involving $2^{1} \pi \pi^{*}, 1^{1} \pi 3 \mathrm{~s} / \pi \sigma^{*}$ and $1^{1} \pi \pi^{*}$. This supports our interpretation of our earlier experimental data ${ }^{43,44}$ and is consistent with the experimental observations of others. ${ }^{32,41,42}$

\section{Computational details}

Ground-state minima and excited state minima and TSs were optimized using the state specific complete active-space selfconsistent field (SS-CASSCF) method. MECIs were optimized using state-averaged CASSCF (SA-CASSCF) ${ }^{46-48}$ with equal weighting given to the two states forming the CI.

The different decay pathways relevant for the photochemistry of aniline were studied using linear interpolation in internal coordinates, relaxed potential scans and minimum energy path (MEP) calculations using the SA-CASSCF method. The MEP calculations were based on the intrinsic reaction coordinate (IRC) method. ${ }^{49,50}$ Although IRC calculations are often started at a TS, the IRC calculation performed in this work (Section 6) is started at the FC geometry using the gradient as the initial relaxation direction.

The CASSCF method is known to describe the multiconfigurational nature of excited state electronic wavefunctions correctly, which is a crucial requirement for systems involving conical intersections, bond breaking or strong geometrical distortions. However, for the relatively small active spaces used in this work, ${ }^{51}$ it does not include dynamical correlation effects and therefore the energies may be inaccurate. To obtain more reliable energies, single-point extended multi-configuration quasidegenerate second-order perturbation theory (XMCQDPT2) calculations ${ }^{52}$ were carried out at optimised stationary points and along decay pathways calculated using the CASSCF method. This protocol, termed MS-MR-PT2//CASSCF, is used because the XMCQDPT2 analytic gradient, required to optimize stationary points efficiently, is not available. This procedure ${ }^{53}$ has been used in previous investigations of the photochemistry of small organic molecule, including DNA bases. ${ }^{54-57}$

The XMCQDPT2 method is a new approach to second order multi-state multi-reference perturbation theory developed by Granovsky. $^{52}$ It is an extension of the MCQDPT2 method $^{58}$ designed to correct for some of the known deficiencies of the latter and other flavours of MS-MR-PT2, such as the problematic behaviour of the energies near conical intersections or avoided crossings. Since its implementation in the Firefly QC package, ${ }^{59}$ the XMCQDPT2 method has been applied to a number of problems of biological and photochemical interest. ${ }^{60-67}$ In addition, it was shown to compare favourably with respect to experimental observations and other high-level theoretical methods. ${ }^{68-70}$

In XMCQDPT2 calculations, it is important to include more CASSCF states in the model space spanned by the zero-order effective Hamiltonian than in the state averaged CASSCF stage. ${ }^{52,71,72}$ In all the XMCQDPT2 calculations reported here, the 30 lowest CASSCF states were included in the model space spanned by the XMCQDPT2 zero-order effective Hamiltonian. Test calculations at the ground state equilibrium and various distorted geometries were performed to check that this size of model space yielded converged energies. An Intruder State Avoidance (ISA) denominator shift of 0.02 was used in all the XMCQDPT2 calculations.

Two different active spaces were employed for the CASSCF calculations. CAS1 consists of 8 electrons in 7 orbitals: 3 occupied $\pi$ orbitals and the corresponding 3 unoccupied $\pi^{*}$ orbitals (in the reference ground-state configuration) together with the occupied nitrogen lone-pair orbital. Since it does not include the $3 \mathrm{~s} / \sigma^{*}$ orbital, this active space cannot describe the ${ }^{1} \pi 3 \mathrm{~s} / \pi \sigma^{*}$ state. CAS2 consists of 10 electrons in 9 orbitals: CAS1 augmented with a pair of $\sigma$ and $3 \mathrm{~s} / \sigma^{*}$ orbitals centered on the amino group. $\dagger$ The $3 \mathrm{~s} / \sigma^{*}$ orbital has strong Rydberg character in the FC region and is needed to describe the low-lying $1^{1} \pi 3 \mathrm{~s} / \pi \sigma^{*}$ state, which is known to play an important role in the photochemistry of aniline and many other substituted aromatic organic compounds. ${ }^{73,74}$ From now on, the $1^{1} \pi 3 \mathrm{~s} / \pi \sigma^{*}$ state will be simply labelled as $1 \pi \sigma^{*}$.

All the calculations performed with the CAS1 active space used the $6-311 \mathrm{G}^{* *}$ basis set whereas the calculations performed 
with the CAS2 active space use the $6-311++\mathrm{G}^{* *}$ basis set augmented with two diffuse $s$ functions and two sets of diffuse $p$ functions on the nitrogen atom as well as a single diffuse $s$ functions on each of the two amino hydrogen atoms. The exponents of the supplementary diffuse functions are determined in an even tempered manner by dividing the exponent of the most diffuse $s$ and $p$ functions already present in the $6-311++G^{* *}$ basis set by a factor of 3.0. Such an extension of the basis set was found to have a significant effect on the $1 \pi \sigma^{*}$ state vertical excitation energy and the height of the barrier to photodissociation, as has been found for pyrrole ${ }^{75,76}$ and phenol. ${ }^{77}$

For the MECI optimizations using the CAS1 active space, we found it necessary to use a quadratically convergent algorithm for the CASSCF wavefunction. The orbital rotation derivative contributions from the coupled perturbed multi-configurational self-consistent field (CP-MCSCF) equations were neglected in the MECI optimizations using the CAS2 active space.

Throughout this paper, the notation SA $n$-CASSCF is used to describe a state averaged CASSCF calculation using orbitals averaged over $n$ electronic states.

The CASSCF optimizations were performed using the Gaussian 03 program package ${ }^{78}$ and the single point CASSCF and XMCQDPT2 calculations were performed using the Firefly QC package, ${ }^{59}$ which is based partially on the GAMESS (US) source code. ${ }^{79}$

\section{Vertical and adiabatic excitation energies}

The equilibrium geometries of the ground and $1^{1} \pi \pi^{*}$ states, optimized using the CAS1 active space, are shown in Fig. 2. Both have $C_{\mathrm{s}}$ symmetry and a pyramidal arrangement around the nitrogen atom.

The focus of this paper is the four lowest lying singlet electronic states, which we label GS, $1 \pi \pi^{*}, 1 \pi \sigma^{*}$ and $2 \pi \pi^{*}$, in order of increasing energy. ${ }^{37,39}$ The vertical excitation energies computed using the two active spaces described in Section 2 are reported in Table 1 and compared with previous calculations and experimental values.

Generally, the calculation of excitation energies using multireference perturbation techniques require that all states of

Table 1 SA-CASSCF and XMCQDPT2 vertical excitation energies, calculated using the two active spaces described in Section 2, compared with values from previous high-level $a b$ initio calculations and experimental values. The theoretical adiabatic excitation energies and experimental 0-0 transition energy of the $1 \pi \pi^{*}$ state are given between parenthesis

\begin{tabular}{lllll}
\hline & $\mathrm{A}^{\prime \prime} 1 \pi \pi^{*}$ & $\mathrm{~A}^{\prime} 1 \pi \sigma^{*}$ & $\mathrm{~A}^{\prime} 2 \pi \pi^{*}$ & $\mathrm{~A}^{\prime \prime} 2 \pi \sigma^{*}$ \\
\hline $\mathrm{CAS1}^{a}$ & $4.81(4.59)$ & - & 7.38 & - \\
$\mathrm{CAS1}^{2}$ XMCQDPT2 $^{a}$ & $4.28(4.02)$ & - & 5.39 & - \\
$\mathrm{CAS2}^{a}$ & $4.81(4.59)$ & 4.90 & 7.36 & 6.19 \\
$\mathrm{CAS}^{2} /$ XMCQDPT2 $^{a}$ & $4.22(3.96)$ & 4.74 & 5.25 & 6.25 \\
SAC-CI $^{37}$ & 4.20 & 4.53 & 5.34 & 6.39 \\
MS-CASPT2 $^{39}$ & 4.33 & 4.85 & 5.54 & 6.28 \\
CR-EOM-CCSD(T) $^{40}$ & 4.21 & 4.69 & 5.42 & - \\
Exp. & $4.41(4.22)^{3}$ & $4.60^{b}$ & $5.42^{3}$ & -
\end{tabular}

${ }^{a}$ This work. ${ }^{b}$ From ref. 38 , assigned as the $0-0$ transition. interest are described uniformly well at the CASSCF level. This requirement often implies the use of state averaged CASSCF orbitals since state specific CASSCF orbitals can be quite different for the different electronic states of interest. This is specially true when the states of interest have a different nature. For instance, Stavros et al. ${ }^{32}$ have shown that the $1 \pi \pi^{*}$ state arises from a local excitation within the phenyl ring whereas the $2 \pi \pi^{*}$ state has a significant charge transfer character. In addition the $1 \pi \sigma^{*}$ state has a significant $3 \mathrm{~s}$ Rydberg character at the FC geometry. In this case, a state specific CASSCF calculation leads to a significant valence-Rydberg mixing whereas a state averaged CASSCF calculation yields orbitals with a well defined character. It was therefore necessary to use state averaged CASSCF orbitals for a well balanced description of the electronic states of interest at the XMCQDPT2 level of theory.

The CAS1 active space can not describe the $1 \pi \sigma^{*}$ state and is used to compute the two first $\pi \pi^{*}$ state excitation energies at the SA3CASSCF and XMCQDPT2 levels of theory. The CASSCF $1 \pi \pi^{*}$ vertical excitation energy is in reasonable agreement with the experimental value. In contrast, the $2 \pi \pi^{*}$ vertical excitation energy is grossly overestimated with an error of $1.96 \mathrm{eV}$. This is not unexpected as the CASSCF method has a well known propensity to overestimate excitation energies to excited states with significant charge transfer character. The XMCQDPT2 calculation using the same CASSCF wavefunction yields excitation energies in good agreement with previous calculations and experimental observations.

In addition to the $1 \pi \sigma^{*}$ state, the CAS2 active space generates a second low-lying $\pi 3 \mathrm{~s} / \pi \sigma^{*}$ state, hereafter denoted $2 \pi \sigma^{*}$, which was calculated at 6.39 and $6.28 \mathrm{eV}$ respectively in previous work. ${ }^{37,39}$ Because of the large overestimation of the $2 \pi \pi^{*}$ state, the CASSCF method wrongly predicts the $2 \pi \sigma^{*}$ state below the $2 \pi \pi^{*}$ state. The correct ordering is however recovered at the XMCQDPT2 level of theory. SA5-CASSCF and XMCQDPT2 vertical excitation energies using the CAS2 active space are presented in Table 1. These results show that the corrections due to the inclusion of the dynamical correlation energy are less important for the $\pi \sigma^{*}$ states than for the $\pi \pi^{*}$ states. Overall, the XMCQDPT2 method provides accurate and well balanced results for the vertical excitation energies of the electronic states of interest.

Finally, we note that the CR-EOM-CCSD(T) calculations of Worth et al. ${ }^{40}$ predicted the $3 \mathrm{p}_{z}$ and $3 \mathrm{p}_{y}$ states to lie at 5.31 and $5.42 \mathrm{eV}$ respectively, between the $1 \pi \sigma^{*}$ and $2 \pi \pi^{*}$ states which is in disagreement with previous high level calculations. ${ }^{37,39}$ We have performed a calculation at the XMCQDPT2 level of theory using the CAS2 active space augmented with the two corresponding $3 p$ Rydberg orbitals. This calculation predicted both states to lie slightly above the $2 \pi \pi^{*}$ state, at 5.58 and $5.62 \mathrm{eV}$ respectively. These states are not considered further in this paper.

\section{Photochemistry after excitation to the $1 \pi \pi^{\star}$ state}

In this section, we consider the decay pathways of aniline after excitation to the $1 \pi \pi^{*}$ state, at energies below the vertical excitation energy of the $1 \pi \sigma^{*}$ state. 
In ref. 32 , the $1 \pi \pi^{*}$ state was described as arising from a local excitation within the phenyl ring, and the analogy with the $S_{1}$ state of benzene was pointed out. Indeed, the photochemistry of aniline after excitation to the $1 \pi \pi^{*}$ state shares many similarities with that of benzene. Following excitation with wavelengths $>245 \mathrm{~nm}$, benzene fluoresces with a quantum yield of approximately 0.2 and the remaining population decays via ISC to a low lying triplet state. These two processes take place on the timescale of 10-100 ns. ${ }^{80,81}$ At wavelengths $<245 \mathrm{~nm}$ (corresponding to $>3000 \mathrm{~cm}^{-1}$ above the $\mathrm{S}_{1} \leftarrow \mathrm{S}_{0} 0-0$ transition) the fluorescence is quenched and the molecule quickly decays to the ground state by internal conversion (IC) via the so-called prefulvene conical intersection. ${ }^{82-90}$ This conical intersection occurs at a geometry characterized by a strong out-of-plane distortion of one of the carbon atoms, accompanied by a strong reduction of the distance between carbon atoms either side of the one raised out of plane to generate the crossing. The activation energy needed to reach the prefulvene deactivation channel has been shown theoretically to be the consequence of the presence of a potential barrier on the pathway connecting the FC region and the MECI on the $S_{1}$ PES. Conical intersections between the first excited $\pi \pi^{*}$ state and the ground state with similar geometries have been described in many other aromatic molecules, for example, pyrazine ${ }^{91}$ and phenol. ${ }^{92}$

In aniline, following excitation to the $1 \pi \pi^{*}$ state at wavelengths $>270 \mathrm{~nm}$, the fluorescence quantum yields are comparable to those of benzene and single vibronic level lifetimes ranging from 3 to $9 \mathrm{~ns}$ have been reported. ${ }^{33,34}$ Whereas in benzene the six carbon atoms are equivalent, the presence of the amino group in aniline breaks this symmetry and the $\mathrm{C} 1$, C2, C3 and C4 carbon atoms (see Fig. 1) are not equivalent. As we show below, this gives rise to the presence of four distinct $\mathrm{S}_{1}\left(1 \pi \pi^{*}\right) / \mathrm{GS}$ prefulvene-like MECI points, labelled $\mathrm{CI}_{1 \pi \pi^{*} / \mathrm{GS}}^{i}$ $(i=1,2,3,4)$, where the superscript refers to the out-of-plane carbon atom (see Fig. 1). Although three of these $\left(\mathrm{CI}_{1 \pi \pi^{*} / \mathrm{GS}}^{1}\right.$, $\mathrm{CI}_{1 \pi \pi^{*} / \mathrm{GS}}^{2}$ and $\mathrm{CI}_{1 \pi \pi^{*} / \mathrm{GS}}^{3}$ ) have been reported before, ${ }^{32}$ to the best of our knowledge this is the first report of $\mathrm{CI}_{1 \pi \pi^{*} / \mathrm{Gs}}^{4}$. It is worth noting that a similar prefulvene-like MECI has been found in phenol $^{92}$ with an out-of-plane distortion of the carbon atom carrying the hydroxyl group. TSs have been found between the $1 \pi \pi^{*}$ equilibrium geometry and $\mathrm{CI}_{1 \pi \pi^{*} / \mathrm{GS}}^{1}$ and $\mathrm{CI}_{1 \pi \pi^{*} / \mathrm{GS}}^{4}$, indicating the presence of potential barriers along these pathways.

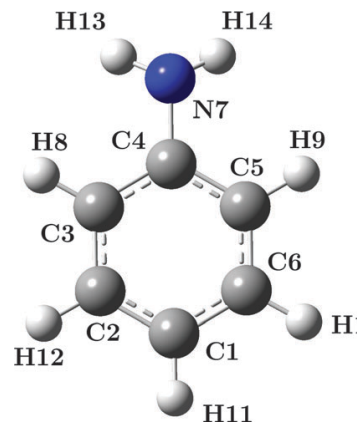

Fig. 1 The aniline molecule with the numbering scheme used throughout this paper.

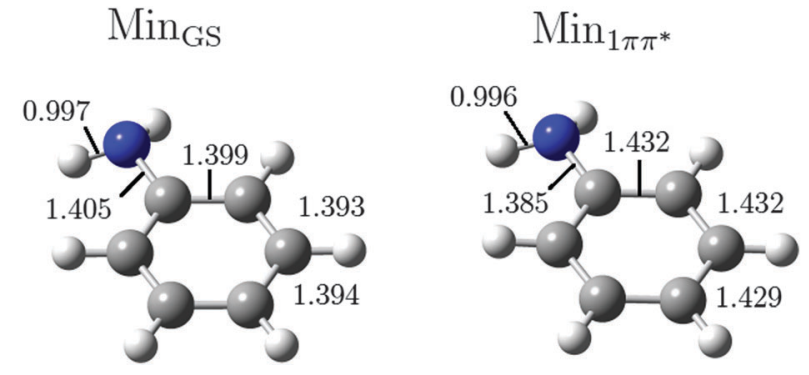

Fig. 2 Ground and $1 \pi \pi^{\star}$ state CASSCF optimized geometries

Potential energy barriers along the pathway to the prefulvene MECI are known in benzene, ${ }^{82-90}$ pyrazine $^{91}$ and phenol. ${ }^{92}$ The four MECI and two TS geometries, optimized using the CAS1 active space are presented in Fig. 3.

No TSs were found on the pathway connecting the $1 \pi \pi^{*}$ equilibrium geometry and $\mathrm{CI}_{1 \pi \pi^{*} / \mathrm{GS}}^{2}$ or $\mathrm{CI}_{1 \pi \pi^{*} / \mathrm{GS}}^{3}$. However, in both cases, the pathway crosses a very flat portion of the $1 \pi \pi^{*}$ PES before reaching the MECI point. Both CASSCF and XMCQDPT2 energies of the four MECIs and two TSs are listed in Table 2. The XMCQDPT2 energies have been computed at the CASSCF optimized geometries. Because the degeneracy is lifted for XMCQDPT2 calculations at CASSCF optimized MECI points, the averaged energies of the two states and the magnitudes of the energy gaps between them are reported.
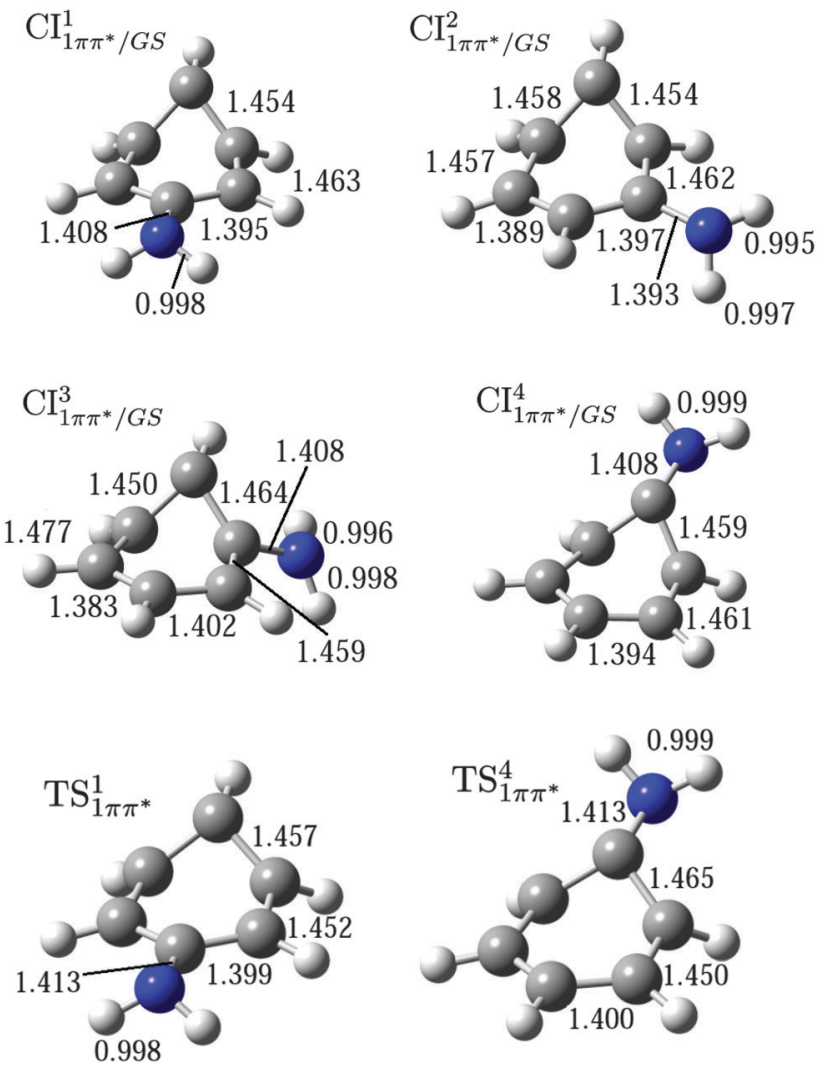

Fig. 3 Geometries of the four prefulvene-like MECIs and of the two TSs, $\mathrm{TS}_{1 \pi \pi^{\star}}^{1}$ and $\mathrm{TS}_{1 \pi \pi^{\star}}^{4}$, optimized along the pathways leading to $\mathrm{Cl}_{1 \pi \pi^{\star} / \mathrm{GS}}^{1}$ and $\mathrm{Cl}_{1 \pi \pi^{\star} / \mathrm{GS}}^{4}$ respectively. Optimizations were performed using the CAS1 active space. 
Table 2 SA2-CASSCF and XMCQDPT2 prefulvene $\mathrm{MECl}$ and TS energies in $\mathrm{eV}$, relative the ground state equilibrium geometry. The XMCQDPT2 $\mathrm{MECl}$ energies are averaged over the GS and $1 \pi \pi^{*}$ states. The values between parenthesis are the magnitude of the energy gap between the two states (see text for details)

\begin{tabular}{lll}
\hline & CASSCF & XMCQDPT2 \\
\hline $\mathrm{CI}_{1 \pi \pi^{*} / \mathrm{GS}}^{1}$ & 5.50 & $4.87(0.22)$ \\
$\mathrm{CI}_{1 \pi \pi^{*} / \mathrm{GS}}^{2}$ & 5.38 & $4.76(0.07)$ \\
$\mathrm{CI}_{1 \pi \pi^{*} / \mathrm{GS}}^{3}$ & 5.39 & $4.69(0.21)$ \\
$\mathrm{CI}_{1 \pi \pi^{*} / \mathrm{GS}}^{4}$ & 5.26 & $4.55(0.46)$ \\
$\mathrm{TS}_{1 \pi \pi^{*}}^{1}$ & 5.57 & 5.10 \\
$\mathrm{TS}_{1 \pi \pi^{*}}^{4}$ & 5.35 & 4.96
\end{tabular}

These results show that, both at the CASSCF and XMCQDPT2 levels of theory, the $\mathrm{CI}_{1 \pi \pi^{*} / \mathrm{GS}}^{4}$ MECI appears at slightly lower energy than the three others. Note that, the dynamical correlation energy correction is smaller at the TSs than at the MECIs. This indicates that the XMCQDPT2 barriers are larger than the CASSCF ones. Therefore, the lack of CASSCF TS on the pathways leading to $\mathrm{CI}_{1 \pi \pi^{*} / \mathrm{GS}}^{2}$ and $\mathrm{CI}_{1 \pi \pi^{*} / \mathrm{GS}}^{3}$ might be a consequence of the non uniform accuracy of the CASSCF method along the pathways.

Fig. 4 presents the pathway between the $1 \pi \pi^{*}$ equilibrium geometry and the $\mathrm{CI}_{1 \pi \pi^{*} / \mathrm{GS}}^{4}$ MECI geometries calculated at the SA2-CASSCF and XMCQDPT2 levels of theory. A single linearly interpolated scan between these two geometries would have overestimated the height of the potential energy barrier separating them so the scan was split into two parts: from the $1 \pi \pi^{*}$ equilibrium geometry to $\mathrm{TS}_{1 \pi \pi^{*}}^{4}$ and from $\mathrm{TS}_{1 \pi \pi^{*}}^{4}$ to the $\mathrm{CI}_{1 \pi \pi^{*} / \mathrm{GS}}^{4}$ MECI geometry.

Our calculation shows that the energy required to open the prefulvene decay channel is higher than the vertical excitation energy of the $1 \pi \sigma^{*}$ state (Table 1 ) and therefore, processes taking place on the $1 \pi \sigma^{*}$ surface are likely to compete with the prefulvene decay channel. Indeed, Stavros et $a l^{32}$ and Montero et $a l^{42}$

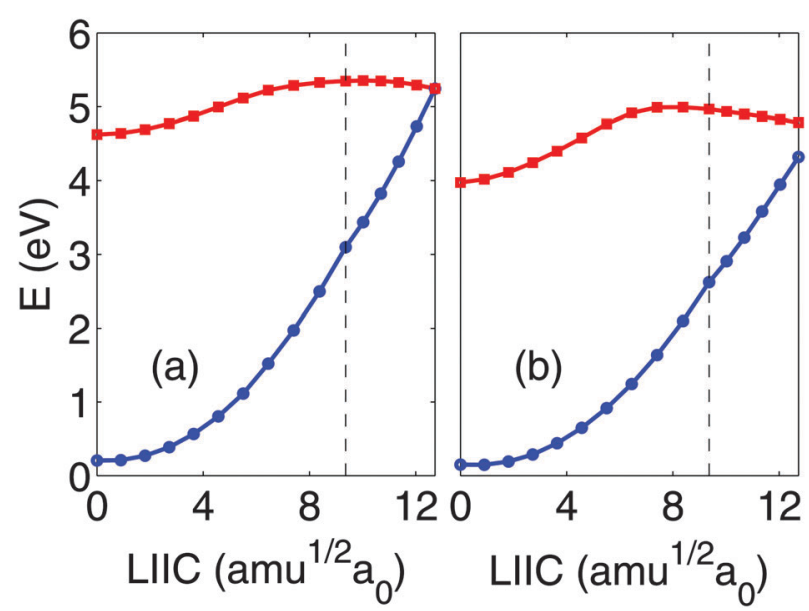

Fig. 4 Ground (blue line with circles) and $1 \pi \pi^{\star}$ (red line with squares) state potential energy profiles along the linearly interpolated internal coordinate (LIIC) from the $1 \pi \pi^{\star}$ equilibrium geometry to the $\mathrm{Cl}_{1 \pi \pi^{*} / \mathrm{GS}}^{4} \mathrm{MECl}$ computed at the (a) SA2-CASSCF and (b) XMCQDPT2 levels of theory. The vertical black dashed line marks the $\mathrm{TS}_{1 \pi \pi^{*}}^{4}$ position (see text for details). reported evidence of decay through the prefulvene channel after excitation of the $2 \pi \pi^{*}$ state. This decay pathway implies evolution on the $2 \pi \pi^{*}$ PES towards a $2 \pi \pi^{*} / 1 \pi \pi^{*}$ CI and subsequent relaxation on the $1 \pi \pi^{*}$ PES towards the prefulvene CI. In this context, the new $\mathrm{CI}_{1 \pi \pi^{*} / \mathrm{GS}}^{4}$ MECI reported here seems particularly relevant since its geometry is similar to the $2 \pi \pi^{*} /$ $1 \pi \pi^{*}$ MECI. Both MECIs involve a strong out-of-plane distortion of the carbon atom carrying the amino group. Therefore, the direct pathway from the $2 \pi \pi^{*} / 1 \pi \pi^{*}$ MECI to the $\mathrm{CI}_{1 \pi \pi^{*} / \mathrm{GS}}^{4} \mathrm{MECI}$ involves much less IVR than the corresponding pathways to the other prefulvene MECIs (see Section 6).

\section{Photochemistry after excitation to the $1 \pi \sigma^{*}$ state}

In this section, we study the decay pathways of aniline following excitation at wavelengths $<269 \mathrm{~nm}$, i.e. above the onset of the $1 \pi \sigma^{*}$ state. $^{38}$

In the FC region, the $1 \pi \sigma^{*}$ state PES has a quasi-bound minimum associated with strong Rydberg $3 \mathrm{~s}$ character on the $\mathrm{N}$ atom. However, along the $\mathrm{N}-\mathrm{H}$ stretching coordinate, the $1 \pi \sigma^{*}$ state acquires a valence $\sigma^{*}$ character and the PES becomes dissociative. The quasi-bound and dissociative components of the PES are separated by a significant barrier, the height of which has been estimated at $0.5 \mathrm{eV}$ using the EOM-CCSD method..$^{40}$ The $1 \pi \sigma^{*}$ state and its role in the electronic relaxation of photoexcited aniline has been the subject of a number of recent investigations. ${ }^{32,40-45}$

We have performed a relaxed potential energy scan along the $\mathrm{N}-\mathrm{H}$ stretching coordinate on the $1 \pi \sigma^{*} \mathrm{PES}$, i.e. at each point, the N7-H14 bond length is kept fixed and the geometry of the rest of the molecule is optimized. The molecule is planar with $C_{2 \mathrm{v}}$ symmetry at the $1 \pi \sigma^{*}$ equilibrium geometry (see Fig. 6). Upon elongation of the N7-H14 bond, the symmetry is reduced to $C_{\mathrm{s}}{ }^{\prime}$. Here, the $C_{\mathrm{s}}{ }^{\prime}$ notation is used to distinguish the planar geometry (with non-equal $\mathrm{N}-\mathrm{H}$ bond lengths), i.e. where the symmetry plane is the molecular plane, from the ground state pyramidal equilibrium geometry of $C_{\mathrm{s}}$ symmetry, where the symmetry plane is perpendicular to the phenyl ring and bisects the $\mathrm{H}-\mathrm{N}-\mathrm{H}$ angle of the amino group. In $C_{\mathrm{s}}{ }^{\prime}$ symmetry, the $1 \pi \sigma^{*}$ state belongs to the $\mathrm{A}^{\prime}$ irreducible representation whereas the GS, $1 \pi \pi^{*}$ and $2 \pi \pi^{*}$ states belong to the $\mathrm{A}^{\prime}$ irreducible representation. In these calculations, the $\mathrm{A}^{\prime}$ and $\mathrm{A}^{\prime \prime}$ states have been computed separately. ${ }^{93}$ The geometries on the $1 \pi \sigma^{*}$ PES are optimized at the SS-CASSCF level of theory using the CAS2 active space. We note that in $C_{\mathrm{s}}{ }^{\prime}$ symmetry, there is no valenceRydberg mixing problem since the valence and Rydberg states have different symmetry. The $\mathrm{A}^{\prime}$ state energies at the optimized geometries have been computed at the SA3-CASSCF level of theory using the same active space. XMCQDPT2 calculations are also performed at the CASSCF optimized geometries and the results of both calculations are presented in Fig. 5 .

Interestingly, although the $1 \pi \sigma^{*}$ state is the second excited state at the FC geometry (see Table 1), from Fig. 5 it is clear that it lies below the $1 \pi \pi^{*}$ state in the CASSCF relaxed scan. This is a 


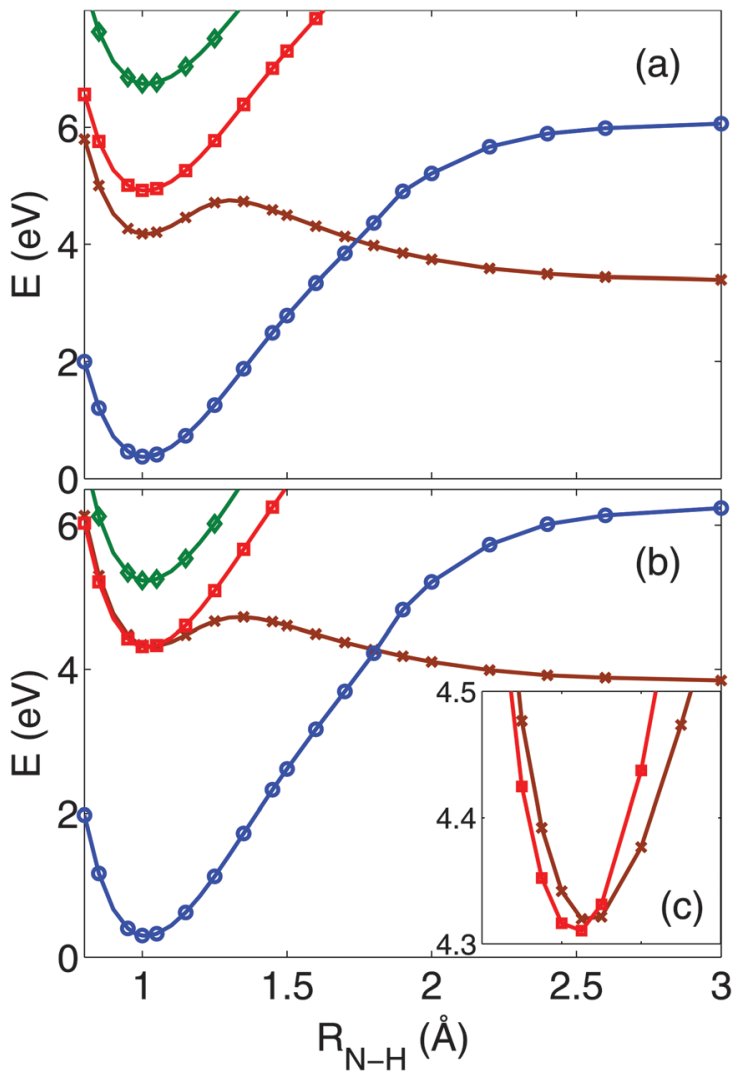

Fig. 5 Relaxed potential energy scan along the $\mathrm{N}-\mathrm{H}$ coordinate, computed at the (a) CASSCF and (b) XMCQDPT2 levels of theory using the CAS2 active space, showing the ground-state (blue circles), $1 \pi \pi^{*}$ state (red squares), $1 \pi \sigma^{\star}$ state (brown crosses), $2 \pi \pi^{\star}$ state (green diamonds). The geometries are optimized on the $1 \pi \sigma^{*}$ state at the SS-CASSCF level of theory using $C_{\mathrm{s}}{ }^{\prime}$ symmetry. A zoom of the $1 \pi \pi^{\star}$ and $1 \pi \sigma^{*}$ states potential scans around the $1 \pi \sigma^{\star}$ minimum is shown in panel (c).
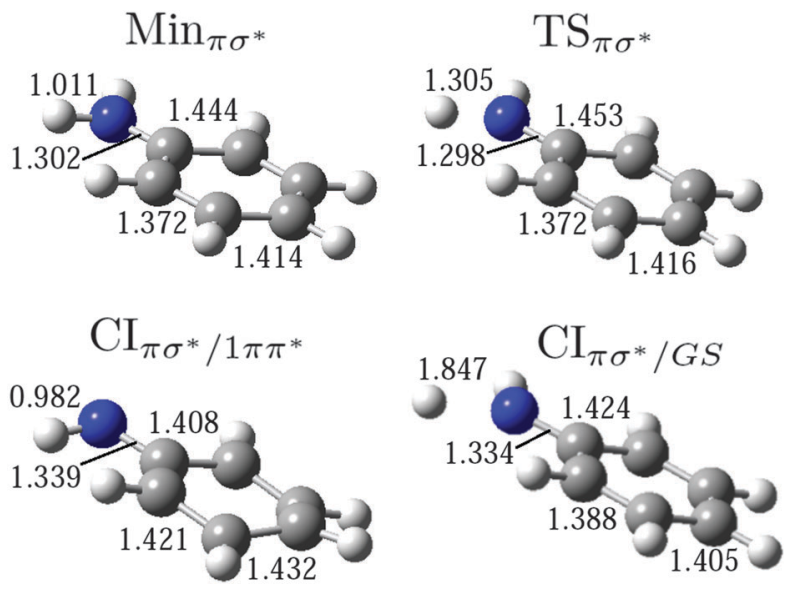

Fig. 6 Geometries of the $1 \pi \sigma^{*}$ state local minimum and TS as well as of the $\pi \sigma^{\star} / \pi \pi^{\star}$ and $\pi \sigma^{\star} / \mathrm{GS}$ MECls.

strong indication that upon relaxation on the $1 \pi \sigma^{*}$ state from the FC geometry, the system must evolve toward a $\pi \sigma^{*} / 1 \pi \pi^{*}$ seam of conical intersections (see Fig. 7 below). At long $\mathrm{N}-\mathrm{H}$ distance, the $1 \pi \sigma^{*}$ curve crosses the GS curve, indicating a

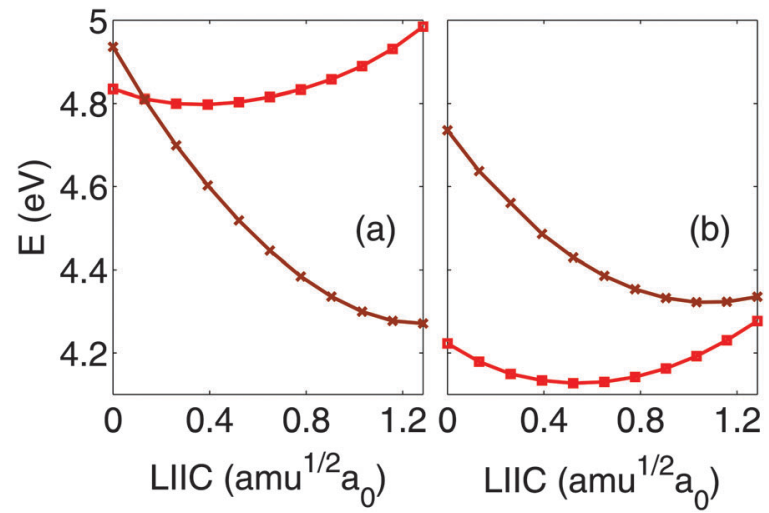

Fig. $71 \pi \pi^{*}$ (red squares) and $1 \pi \sigma^{*}$ (brown crosses) potential energy curves along the linearly interpolated internal coordinate between the FC and CASSCF optimized $\mathrm{Min}_{\pi \sigma^{*}}$ geometries, computed at the (a) SA5-CASSCF and (b) XMCQDPT2 levels of theory.

competition between internal conversion to the ground state and $\mathrm{N}-\mathrm{H}$ dissociation upon evolution on the dissociative part of the $1 \pi \sigma^{*}$ state. Both $\pi \sigma^{*} / 1 \pi \pi^{*}$ and $\pi \sigma^{*} /$ GS MECIs have been reported previously. ${ }^{32}$ Our CASSCF calculation yields a height of $0.58 \mathrm{eV}$ for the barrier separating the quasi-bound well from the dissociative part on the $1 \pi \sigma^{*}$ curve, and an $\mathrm{N}-\mathrm{H}$ bond strength of $3.37 \mathrm{eV}$, which is a significant underestimate compared to the experimental value of $3.92 \mathrm{eV}$ reported by Ashfold and coworkers. ${ }^{41}$ The CASSCF optimized geometries of the $1 \pi \sigma^{*}$ state local minimum and TS, noted $\operatorname{Min}_{\pi \sigma^{*}}$ and $\mathrm{TS}_{\pi \sigma^{*}}$ respectively, as well as of the $\pi \sigma^{*} / \pi \pi^{*}$ and $\pi \sigma^{*} /$ GS MECIs, noted $\mathrm{CI}_{\pi \sigma^{*} / 1 \pi \pi^{*}}$ and $\mathrm{CI}_{\pi \sigma^{*} / \mathrm{GS}}$ respectively, are presented in Fig. 6 .

At the $\operatorname{Min}_{\pi \sigma^{*}}$ geometry, the molecule is planar with a $C_{2 \mathrm{v}}$ symmetry point group and an $\mathrm{N}-\mathrm{H}$ bond length of $1.01 \AA$. The $\mathrm{TS}_{\pi \sigma^{*}}$ and $\mathrm{CI}_{\pi \sigma^{*} / \mathrm{GS}}$ geometries are also planar, with $C_{\mathrm{s}}{ }^{\prime}$ symmetry and $\mathrm{N}-\mathrm{H}$ bond lengths of $1.31 \AA$ and $1.85 \AA$, respectively.

We now discuss the results obtained from the XMCQDPT2 calculation of the relaxed scan at the CASSCF optimized geometries, shown in Fig. 5(b). We obtain a barrier height of $0.41 \mathrm{eV}$, which is lower than the CASSCF value, and an $\mathrm{N}-\mathrm{H}$ bond strength of $3.83 \mathrm{eV}$, in good agreement with the experimental value of $3.92 \mathrm{eV} .^{41}$ As shown in Section 3, the inclusion of dynamical electron correlation has a greater effect on the $1 \pi \pi^{*}$ state than on the $1 \pi \sigma^{*}$ state and for short N-H distances, the $1 \pi \pi^{*}$ and $1 \pi \sigma^{*}$ states are almost degenerate with the $1 \pi \pi^{*}$ state lying slightly below the $1 \pi \sigma^{*}$ state. As the $\mathrm{N}-\mathrm{H}$ distance increases slightly, the $1 \pi \sigma^{*}$ crosses the $1 \pi \pi^{*}$ curve close to its local minimum in the quasi-bound well. This supports the conclusion drawn from experimental observation of the relaxation dynamics following excitation close to or above the minimum of the $1 \pi \sigma^{*}$ state. $^{43,44}$ This has interesting consequences regarding the vibronic structure of the molecule in this range of energies. In ref. 38, an experimental excitation energy of $4.60 \mathrm{eV}$ was attributed to the $0-0$ transition to the $1 \pi \sigma^{*}$ state. However, a conical intersection lying close to the $1 \pi \sigma^{*}$ local minimum points towards the existence of strongly vibronically coupled levels that cannot be attributed easily to vibrational levels of either $1 \pi \pi^{*}$ or $1 \pi \sigma^{*}$ states, as discussed in ref. 94 . 
In order to gain further insight into the competition between relaxation to the $1 \pi \pi^{*}$ state and relaxation to the dissociative part of the $1 \pi \sigma^{*}$ state, we have computed a linearly interpolated scan from the FC to the CASSCF optimized $\operatorname{Min}_{\pi \sigma^{*}}$ geometries (Fig. 7). State averaging over the five lowest states is used in this calculation. At the CASSCF level of theory, the $\pi \sigma^{*} /$ $1 \pi \pi^{*}$ CI appears very close to the FC geometry. However, the situation is quite different at the XMCQDPT2 level of theory and the CI appears at a geometry much closer to the $\operatorname{Min}_{\pi \sigma^{*}}$ geometry. This suggests that, after excitation to the $1 \pi \sigma^{*}$ state, the system will relax toward the $\mathrm{CI}_{\pi \sigma^{*} / 1 \pi \pi^{*}}$, where it will either be transferred to the $1 \pi \pi^{*}$ state and then decay on a very long (nanosecond) timescale, as discussed in the previous section, or stay on the $1 \pi \sigma^{*}$ state and evolve on the dissociative portion of the PES.

\section{Photochemistry after excitation to the $2 \pi \pi^{*}$ state}

In this section, we study the decay pathway of aniline following excitation to the $2 \pi \pi^{*}$ state. Experimentally, this corresponds to excitation with wavelengths $<240 \mathrm{~nm}$. A number of experimental studies have been directed towards unravelling $2 \pi \pi^{*}$ electronic relaxation using a variety of techniques: timeresolved photoelectron spectroscopy, ${ }^{43,44}$ time-resolved photoionisation spectroscopy, ${ }^{42}$ time-resolved velocity-map imaging ${ }^{32}$ and $\mathrm{H}$-atom (Rydberg) photofragment translational spectroscopy. ${ }^{41}$ In all these studies, ultrafast decays were observed, or inferred, and interpreted as either internal conversion to the ground state or $\mathrm{H}$-atom loss on the $1 \pi \sigma^{*}$ surface. For example, Ashfold and coworkers recorded bi-modal total kinetic energy release (TKER) spectra at various excitation wavelengths in the range $240-220 \mathrm{~nm} .^{41}$ The fast $\mathrm{H}$-atom component was attributed to dissociation on the $1 \pi \sigma^{*}$ surface after internal conversion via a $2 \pi \pi^{*} / \pi \sigma^{*} \mathrm{CI}$, or a $2 \pi \pi^{*} / 1 \pi \pi^{*} \mathrm{CI}$ with subsequent transfer to the $1 \pi \sigma^{*}$ surface via the $\pi \sigma^{*} / 1 \pi \pi^{*}$ CI. The slow H-atom component was attributed to dissociation from the ground-state following internal conversion from the $2 \pi \pi^{*}$ state to the ground-state. Montero et al. recorded three different time constants in the same wavelength range, $\tau_{0}=21 \pm 5 \mathrm{fs}, \tau_{1}=150 \pm 30 \mathrm{fs}$ and $\tau_{2}=45 \pm 2$ ps. ${ }^{42}$ The $\tau_{0}$ constant was attributed to an internal conversion through a $2 \pi \pi^{*} / 1 \pi \pi^{*} \mathrm{CI}$, with a subsequent competition between internal conversion to the $1 \pi \sigma^{*}$ state and dissociation, corresponding to the $\tau_{1}$ constant, or internal conversion to the ground state via the prefulvene CI discussed in Section 4, corresponding to the much larger $\tau_{2}$ constant. This large time constant would be the sign of a considerable intramolecular vibrational energy redistribution (IVR) in the $1 \pi \pi^{*}$ state competing with internal conversion to the ground state.

In terms of previous theoretical studies, a $2 \pi \pi^{*} / 1 \pi \pi^{*}$ MECI was reported, ${ }^{32}$ at a geometry involving a strong out-of-plane deformation of the molecule.

Here, we report evidence for a new decay pathway leading to internal conversion to the ground state, mediated by three successive MECIs, two of which have not been reported before.

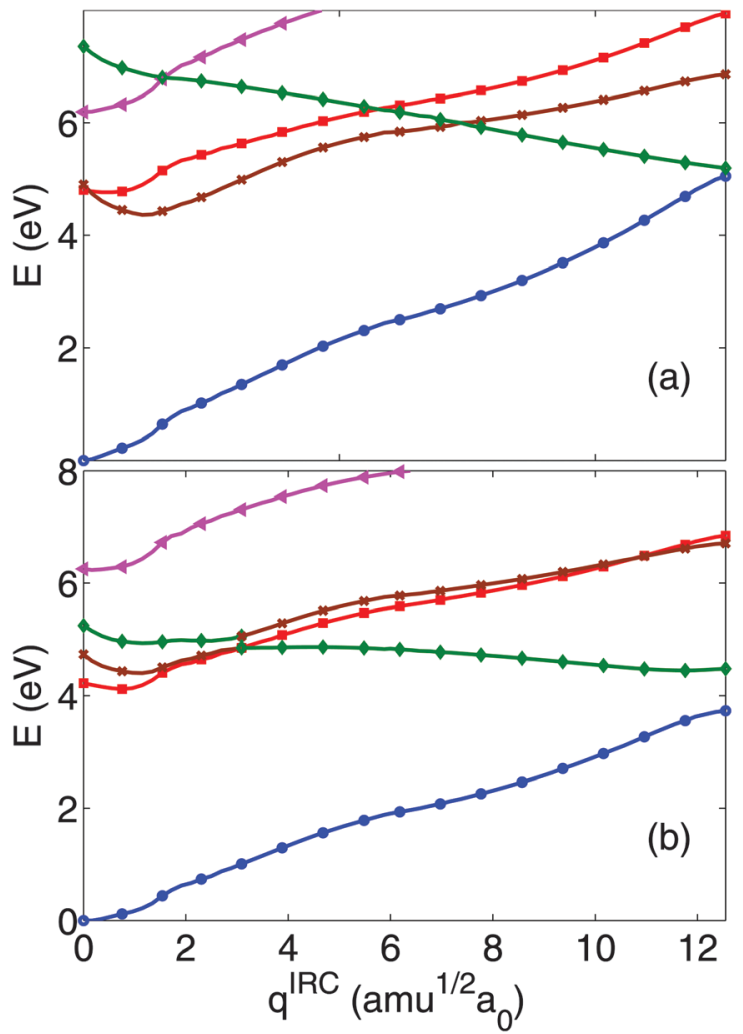

Fig. $8 A^{\prime}$ Ground state (blue circles), $A^{\prime \prime} 1 \pi \pi^{*}$ (red squares), $A^{\prime} 1 \pi \sigma^{*}$ (brown crosses), $A^{\prime} 2 \pi \pi^{\star}$ (green diamonds) and $A^{\prime \prime} 2 \pi \sigma^{\star}$ (magenta triangles) potential energy profiles computed at the SA5-CASSCF (a) and XMCQDPT2 (b) levels of theory along the IRC scan on the $A^{\prime} 2 \pi \pi^{\star}$ state.

The MEP connecting the $2 \pi \pi^{*}$ state at the FC geometry to the ground state is presented in Fig. 8. The MEP calculation was performed in several steps and the geometry was constrained to $C_{\mathrm{s}}$ symmetry. We began by performing an IRC calculation, starting at the FC geometry on the $2 \pi \pi^{*}$ state, using the CAS1 active space and a CASSCF wavefunction averaged over the $1 \pi \pi^{*}$ and $2 \pi \pi^{*}$ states. The initial relaxation direction was taken to be the gradient on the $2 \pi \pi^{*}$ surface. The CAS1 active space is used to filter out the $\pi \sigma^{*}$ states, which greatly simplifies the IRC calculation. The IRC calculation was stopped at the $1 \pi \pi^{*} / 2 \pi \pi^{*}$ crossing. Then, a single point was calculated at a geometry obtained by extrapolating the last IRC step. At this point, we checked that the $2 \pi \pi^{*}$ state was lying below the $1 \pi \pi^{*}$ state. Because the geometry was constrained to $C_{\mathrm{s}}$ symmetry, the symmetry of the $1 \pi \pi^{*}$ and $2 \pi \pi^{*}$ states ( $\mathrm{A}^{\prime \prime}$ and $\mathrm{A}^{\prime}$ respectively) is conserved along the MEP. Therefore, the $1 \pi \pi^{*}$ and $2 \pi \pi^{*}$ states can be distinguished unambiguously by checking their respective wavefunctions. A second IRC calculation was then performed starting at this last geometry, using the CAS1 active space and a CASSCF wavefunction averaged over the $2 \pi \pi^{*}$ and ground states.

In the second stage, in order to include the $\pi \sigma^{*}$ states in our calculations, we performed single point SA-CASSCF and XMCQDPT2 calculations at the geometries obtained from the IRC calculations, using the CAS2 active space. The CASSCF wavefunction was averaged over the five lowest states. The results of these CASSCF and XMCQDPT2 calculations are displayed in 

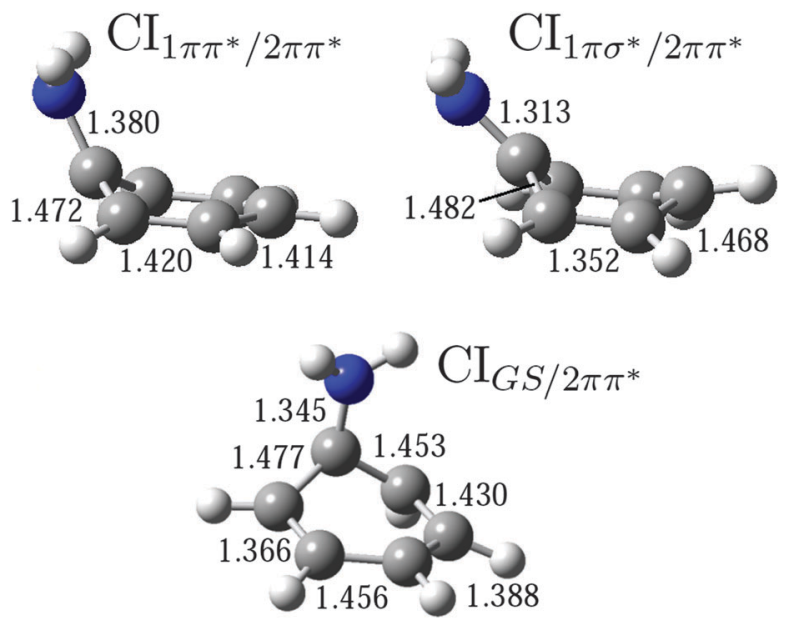

Fig. 9 Geometries of the three MECls involving the $2 \pi \pi^{\star}$ state.

Fig. 8(a) and (b), respectively. From now on, the intrinsic reaction coordinate will be noted $q^{\mathrm{IRC}}$.

First, we consider the CASSCF IRC scan shown in Fig. 8(a). As discussed in Section 3, the CASSCF method yields the wrong state ordering at the FC geometry, i.e. the $2 \pi \sigma^{*}$ state lies below the $2 \pi \pi^{*}$ state. As relaxation on $2 \pi \pi^{*}$ begins from the FC geometry, the $2 \pi \pi^{*}$ state energy decreases and the $2 \pi \sigma^{*}$ state energy increases until the two curves cross around $q^{\mathrm{IRC}}=1.8$. This $2 \pi \pi^{*} / 2 \pi \sigma^{*}$ CI is an artifact of the CASSCF method. Overall, the CASSCF MEP shows the existence of a barrierless relaxation pathway involving a strong out-of-plane deformation of the molecule, resulting in a boat conformation of the phenyl ring at the end of the IRC scan. Along this pathway, the $2 \pi \pi^{*}$ state crosses, successively, the $1 \pi \pi^{*}, 1 \pi \sigma^{*}$ and ground electronic states.

We now discuss the XMCQDPT2 MEP computed at the CASSCF optimized geometries shown in Fig. 8. As already discussed, the correction to the energies from the inclusion of dynamical electron correlation is quite large for the $2 \pi \pi^{*}$ state. Upon relaxation, the energies of the $1 \pi \pi^{*}, 1 \pi \sigma^{*}$ and $2 \pi \pi^{*}$ states come close to one other around $q^{\mathrm{IRC}}=3$. In this region, the three states lie within $0.25 \mathrm{eV}$ of each other, which is a strong indication for the presence of a nearby three-state CI. ${ }^{95-97}$ In contrast to the CASSCF MEP, here the $1 \pi \sigma^{*}$ and $2 \pi \pi^{*}$ states, both of $\mathrm{A}^{\prime}$ symmetry, form a narrowly avoided crossing rather than a true CI. This is, however, just an indication that the XMCQDPT2 pathway passes very close to the $1 \pi \sigma^{*} / 2 \pi \pi^{*}$ CI seam rather than crossing it as in the CASSCF calculation. The pseudo-diabatic assignment used to distinguish between the states is of little significance in the interaction region because the electronic characters of the $1 \pi \sigma^{*}$ and $2 \pi \pi^{*}$ states are mixed; however, as we move away from the three-state CI region, the electronic character of both states become clear again. The same is true for the region where the $2 \pi \pi^{*}$ state approaches the ground state at the end of the scan.

These calculations have unveiled three CIs that are relevant to the electronic relaxation of aniline following excitation to the $2 \pi \pi^{*}$ state: $1 \pi \sigma^{*} / 2 \pi \pi^{*}, 1 \pi \pi^{*} / 2 \pi \pi^{*}$ and GS/ $2 \pi \pi^{*}$ CIs. We have

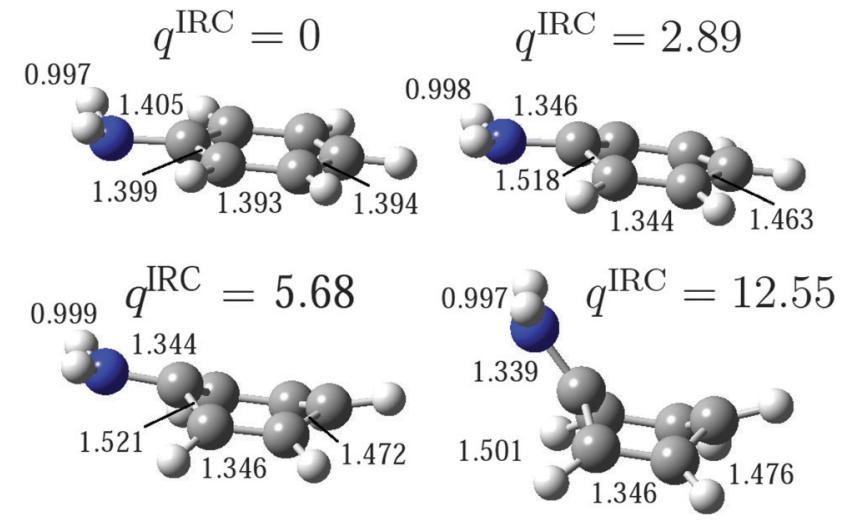

Fig. 10 Geometries at key points along the MEP (see text for details).

optimized the three corresponding MECIs, labelled $\mathrm{CI}_{1 \pi \sigma^{*} / 2 \pi \pi^{*}}$, $\mathrm{CI}_{1 \pi \pi^{*} / 2 \pi \pi^{*}}$ and $\mathrm{CI}_{\mathrm{GS} / 2 \pi \pi^{*}}$, at the CASSCF level of theory using the CAS2 active space for $\mathrm{CI}_{1 \pi \sigma^{*} / 2 \pi \pi^{*}}$ and the CAS1 space for $\mathrm{CI}_{1 \pi \pi^{*} / 2 \pi \pi^{*}}$ and $\mathrm{CI}_{\mathrm{GS} / 2 \pi \pi^{*}}$. The three resulting geometries are shown in Fig. 9. Although $\mathrm{CI}_{1 \pi \pi^{*} / 2 \pi \pi^{*}}$ has been reported previously, ${ }^{32}$ $\mathrm{CI}_{1 \pi \sigma^{*} / 2 \pi \pi^{*}}$ and $\mathrm{CI}_{2 \pi \pi^{*} / \mathrm{GS}}$ are reported here for the first time.

In Fig. 10, we show the geometries of the molecule for four key points along the MEP: the FC geometry $\left(q^{\mathrm{IRC}}=0\right)$, the geometry of the approximate three-state CI $\left(q^{\mathrm{IRC}}=2.89\right)$ the geometry of the $1 \pi \pi^{*} / 2 \pi \pi^{*}$ crossing $\left(q^{\mathrm{IRC}}=5.68\right)$ and the geometry corresponding to the GS $/ 2 \pi \pi^{*}$ crossing $\left(q^{\mathrm{IRC}}=12.55\right)$.

Overall, the XMCQDPT2 decay pathway on the $2 \pi \pi^{*}$ state surface connecting the equilibrium geometry to the $\mathrm{CI}_{2 \pi \pi^{*} / \mathrm{GS}}$ is barrierless, although flatter than the CASSCF computed pathway. This suggests that the corrections due to the inclusion of dynamical electron correlation are greater in the $\mathrm{FC}$ region than in the region of the $\mathrm{CI}_{2 \pi \pi^{*} / \mathrm{GS}}$ corresponding to a strongly distorted geometry. However, another possible explanation is that the geometries along the pathway are obtained via CASSCF partial optimizations, therefore they may be far from the true XMCQDPT2 MEP. Nonetheless, it is interesting to note that similar ring puckering decay pathways have been described in other simple aromatic organic molecules such as pyrrole ${ }^{98}$ or the purine DNA bases adenine ${ }^{55,99}$ and guanine. ${ }^{57}$ Moreover, the boat conformation of the phenyl ring, found at $q^{\mathrm{IRC}}=12.55$ (see Fig. 10), is reminiscent of the Dewar form of benzene, which is know to be populated after excitation to the $2 \pi \pi^{*}$ state of benzene. ${ }^{100-102}$ To investigate this further, we extrapolated from the CI at $q^{\mathrm{IRC}}=12.55$, along the IRC until a geometry where the $2 \pi \pi^{*}$ state is the ground state was found and then performed a geometry optimization. The resulting local minimum on the ground state PES $\left(\operatorname{Min}_{\text {Dew }}\right)$ is an aniline analogue of the Dewar benzene isomer (Fig. 11). We have also located a saddle point $\left(\mathrm{TS}_{\text {Dew }}\right)$ connecting this local minimum to the global equilibrium geometry (Fig. 11).

The energies of the Dewar minimum and TS relative to the global minimum energy, computed at the SA2-CASSCF and XMCQDPT2 level of theory using the CAS1 active space, are presented in Table 3. The SA2-CASSCF energies of the $\mathrm{CI}_{2 \pi \pi^{*} / \mathrm{GS}}$ points, are also reported. They show that the Dewar form of aniline is associated with a local minimum on the ground 

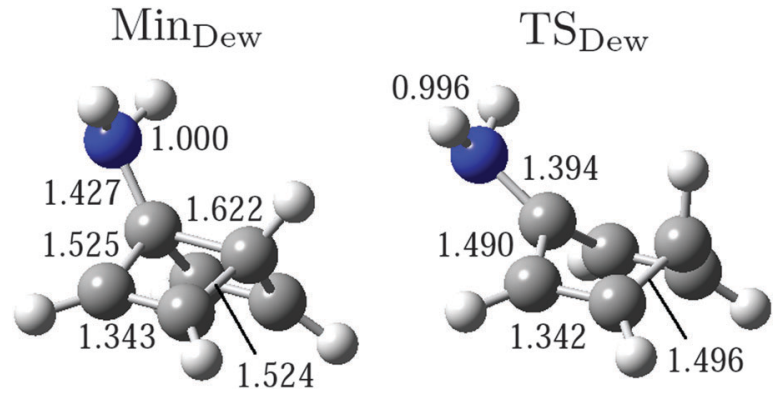

Fig. 11 Geometries of the Dewar minimum and TS on the ground state PES.

Table 3 SA2-CASSCF and XMCQDPT2 energies of the Dewar minimum and TS and SA2-CASSCF energies of the $\mathrm{Cl}_{2 \pi \pi^{*} / G S}$ points. $\mathrm{Cl}_{2 \pi \pi^{*} / \mathrm{GS}}^{\mathrm{MECl}}$ refers to the $\mathrm{MECl}$ (see Fig. 9) and $\mathrm{Cl}_{2 \pi \pi^{\star} / \mathrm{GS}}^{\mathrm{RC}}$ to the $\mathrm{Cl}$ point reached on the IRC path at $q^{\mathrm{IRC}}=12.55$ (see Fig. 8 and 10 )

\begin{tabular}{lll}
\hline & SA2-CASSCF & XMCQDPT2 \\
\hline $\mathrm{Min}_{\text {Dew }}$ & 3.97 & 3.54 \\
$\mathrm{TS}_{\text {Dew }}$ & 4.60 & 4.11 \\
$\mathrm{CI}_{2 \pi \pi^{*} / \mathrm{GS}}^{\mathrm{MECI}}$ & 4.79 & - \\
$\mathrm{CI}_{2 \pi \pi^{*} / \mathrm{GS}}^{\mathrm{RC}}$ & 5.21 & -
\end{tabular}

state PES, separated from the global minimum by a barrier with a height evaluated at $0.57 \mathrm{eV}$. Interestingly, the Dewar form of aniline might provide a possible experimental probe of the ring puckering decay pathway discussed above, i.e. an experiment aiming at its detection after excitation to the $2 \pi \pi^{*}$ state would provide a test of the relevance of this decay path in the photochemistry of aniline.

In addition to the ring-puckering decay pathway discussed above, the potential energy curves in Fig. 8 suggest two alternative decay pathways. At the three-state CI, the molecule can relax to the $1 \pi \pi^{*}$ or $1 \pi \sigma^{*}$ states. In both cases, it is clear from Fig. 8 that the molecule can then relax directly to the FC region. From the FC region, the molecule can then decay to the ground state via the prefulvene $\mathrm{CI}_{1 \pi \pi^{*} / \mathrm{GS}}$ shown in Fig. 3 or the $\mathrm{CI}_{\pi \sigma^{*} / \mathrm{GS}}$ shown in Fig. 6, or dissociate on the $1 \pi \sigma^{*}$ PES. In order to find out if other direct decay pathways to the ground state exist, we performed linearly interpolated scans from the $\mathrm{CI}_{1 \pi \pi^{*} / 2 \pi \pi^{*}}$ to the prefulvene $\mathrm{CI}_{1 \pi \pi^{*} / \mathrm{GS}}$ and from the $\mathrm{CI}_{1 \pi \sigma^{*} / 2 \pi \pi^{*}}$ to the $\mathrm{CI}_{1 \pi \sigma^{*} / \mathrm{GS}}$ respectively, using the CAS2 active space. For the former, the orbitals were averaged over the five lowest states while for the latter, the inclusion of a sixth state was found necessary for a well balanced description of the states of interest along the pathway. Both calculations are presented in Fig. 12.

In both cases, the initial geometries are not taken to be the MECI structures of $\mathrm{CI}_{1 \pi \pi^{*} / 2 \pi \pi^{*}}$ and $\mathrm{CI}_{1 \pi \sigma^{*} / 2 \pi \pi^{*}}$, but rather the CI points reached in the CASSCF IRC pathway of Fig. 8(a). The scan in Fig. 12(a) shows a rather flat but barrierless pathway connecting the $\mathrm{CI}_{1 \pi \pi^{*} / 2 \pi \pi^{*}}$ with the prefulvene $\mathrm{CI}_{1 \pi \pi^{*} / \mathrm{GS}}$. Interestingly, a similar barrierless pathway connecting the $\mathrm{S}_{2} / \mathrm{S}_{1} \mathrm{CI}$ to the prefulvene $\mathrm{S}_{1} / \mathrm{S}_{0} \mathrm{CI}$ in benzene has been calculated. ${ }^{103,104}$ However, Fig. 8(a) shows that the path connecting the $\mathrm{CI}_{1 \pi \pi^{*} / 2 \pi \pi^{*}}$ with the FC geometry is steeper. Therefore, it seems most
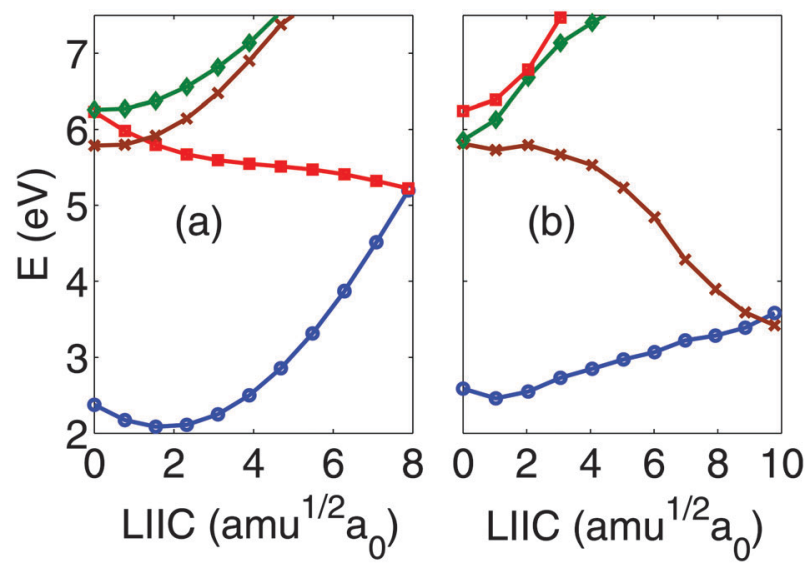

Fig. 12 Linearly interpolated scans (a) from the $\mathrm{Cl}_{1 \pi \pi^{*} / 2 \pi \pi^{*}}$ to the prefulvene $\mathrm{Cl}_{1 \pi \pi^{*} / \mathrm{GS}}$ and (b) from the $\mathrm{Cl}_{1 \pi \sigma^{*} / 2 \pi \pi^{*}}$ to the $\mathrm{Cl}_{1 \pi \sigma^{*} / \mathrm{GS}}$ computed at the SA5-CASSCF and SA6-CASSCF level of theory, respectively, using the CAS2 active space.

likely that, if the molecule relaxes to the $1 \pi \pi^{*}$ state, it will then relax to the FC region where it can relax further via the prefulvene $\mathrm{CI}_{1 \pi \pi^{*} / \mathrm{GS}}$ or via the $\mathrm{N}-\mathrm{H}$ dissociation path on the $1 \pi \sigma^{*}$ state after crossing the $\mathrm{CI}_{\pi \sigma^{*} / 1 \pi \pi^{*}}$, as has been proposed previously. ${ }^{41,42}$

The scan of Fig. 12(b) shows a steep pathway connecting the $\mathrm{CI}_{1 \pi \sigma^{*} / 2 \pi \pi^{*}}$ to the $\mathrm{CI}_{1 \pi \sigma^{*} / \mathrm{GS}}$, suggesting the possibility of very efficient relaxation to the ground state or dissociation on the $1 \pi \sigma^{*}$ PES. A barrier is seen on the $1 \pi \sigma^{*}$ potential energy profile. However this barrier appears much smaller than the barrier on the relaxed $\mathrm{N}-\mathrm{H}$ potential scan shown in Fig. 5, and should therefore be easily overcome by the wavepacket upon relaxation on the $1 \pi \sigma^{*}$ state after crossing the $\mathrm{CI}_{1 \pi \sigma^{*} / 2 \pi \pi^{*}}$.

It is not possible from this work to infer which decay pathway will be preferred by the molecule, after excitation to the $2 \pi \pi^{*}$ state. It is known that nuclear dynamics around CIs do not only depend on the location of the MECI point but also on the topography of the extended CI seam. Specifically, different decay pathways can be preferred depending on which region of the CI seam is reached. ${ }^{105-107}$ Therefore, an extended mapping of the various CI seams connecting the electronic states of interest in this work would provide valuable information on the competition between the different accessible decay channels. Direct dynamics techniques ${ }^{108,109}$ can also be used to follow the relaxation pathway of the molecule directly. The conclusions drawn in this work indicate that both approaches should use post-CASSCF electronic structure calculations to reach the level of accuracy necessary for a reliable description of the relaxation of aniline after electronic excitation.

\section{Summary}

CASSCF and XMCQPDT2 calculations have been employed to determine key geometries and pathways on the potential energy landscape of the four lowest lying electronic singlet states of aniline, $\mathrm{S}_{0}(\mathrm{GS}), 1 \pi \pi^{*}, 1 \pi \sigma^{*}$ and $2 \pi \pi^{*}$. We have located four 
prefulvene-like MECIs connecting the $1 \pi \pi^{*}$ state with GS. The lowest energy MECI, in which the carbon-atom carrying the amino group is distorted out-of-plane, is reported here for the first time. It seems likely that this MECI is involved in nonradiative decay from $1 \pi \pi^{*}$ to the ground-state, although the energy of this MECI lies above the vertical excitation energy to $1 \pi \sigma^{*}$ and will only be accessible at higher excitation energies. We have found a MECI connecting $1 \pi \sigma^{*}$ and $1 \pi \pi^{*}$ states close to the local minimum on $1 \pi \sigma^{*}$. We determine that excitation to $1 \pi \sigma^{*}$ is likely to be followed by relaxation to this MECI where population will subsequently be transferred both to $1 \pi \pi^{*}$ (as observed in our earlier work ${ }^{43,44}$ ) and to the dissociative component of $1 \pi \sigma^{*}$ (as observed by Stavros et al. ${ }^{32}$ ). We also find evidence for a new decay pathway connecting $2 \pi \pi^{*}$ and the ground-state that passes through a three-state CI involving $2 \pi \pi^{*}, 1 \pi \sigma^{*}$ and $1 \pi \pi^{*}$. From this three-state CI, population may be transferred on the lower portion of the $2 \pi \pi^{*}$ state with subsequent relaxation towards the $\mathrm{CI}_{2 \pi \pi^{*} / \mathrm{GS}}$, or on the $1 \pi \sigma^{*}$ or $1 \pi \pi^{*}$ states, with subsequent relaxation towards the $\mathrm{CI}_{1 \pi \sigma^{*} / \mathrm{GS}}$ or prefulvene $\mathrm{CI}_{1 \pi \pi^{*} / \mathrm{GS}}$, respectively. This scheme supports our interpretation of our earlier data ${ }^{43,44}$ and is consistent with the observations of others. ${ }^{32,41,42}$ Overall, our calculations are in agreement with all experimental observations.

\section{Acknowledgements}

Calculations were performed using HPC resources from DSICCUB (Université de Bourgogne). The authors acknowledge support from the European Marie Curie Initial Training Network Grant No. GA-ITN-214962-FASTQUAST for research funding. M. S. and S. G. acknowledge support from the Conseil Régional de Bourgogne. The authors are grateful to G. A. Worth and M. A. Robb for their comments on the manuscript.

\section{Notes and references}

1 K. Kimura, H. Tsubomura and S. Nagakura, Bull. Chem. Soc. Jpn., 1964, 37, 1336.

2 K. Kimura and S. Nagakura, Mol. Phys., 1965, 9, 117.

3 B. N. Rajasekhar, A. Veeraiah, K. Sunanda and B. N. Jagatap, J. Chem. Phys., 2013, 139, 064303.

4 D. G. Lister, J. K. Tyler, J. H. Hog and N. W. Larsen, J. Mol. Struct., 1974, 23, 253.

5 M. Fukuyo, K. Hirotsu and T. Higuchi, Acta Crystallogr., Sect. B: Struct. Crystallogr. Cryst. Chem., 1982, 38, 640.

6 G. Roussy and A. Nonat, J. Mol. Spectrosc., 1986, 118, 180.

7 G. Schultz, G. Portalone, F. Ramondo, A. Domenicano and I. Hargittai, Struct. Chem., 1996, 7, 59.

8 A. Wolf, U. Voets and H.-H. Schmidtke, Theor. Chim. Acta, 1980, 54, 229.

9 Z. Niu, K. M. Dunn and J. E. Boggs, Mol. Phys., 1985, 55, 421. 10 J. E. Boggs and Z. Niu, J. Comput. Chem., 1985, 6, 46.

11 C. W. Bock, P. George and M. Trachtman, Theor. Chim. Acta, 1986, 69, 235.

12 A.-D. Gorse and M. Pesquer, THEOCHEM, 1993, 281, 21.
13 Y. Wang, S. Saebo and C. U. Pittman Jr, THEOCHEM, 1993, 281, 91.

14 M. Castellà-Ventura and E. Kassab, Spectrochim. Acta, Part A, 1994, 50, 69.

15 J. Sponer and P. Hobza, Int. J. Quantum Chem., 1996, 57, 959.

16 O. Bludský, J. Sponer, J. Leszczynski, V. Spirko and P. Hobza, J. Chem. Phys., 1996, 105, 11042.

17 I. López-Tocón, J. C. Otero, M. Becucci, G. Pietraperzia and E. Castellucci, Chem. Phys., 1999, 249, 113.

18 M. A. Palafox, J. L. N. nez and M. Gil, THEOCHEM, 2002, 593, 101.

19 P. M. Wojciechowski, W. Zierkiewicz, D. Michalska and P. Hobza, J. Chem. Phys., 2003, 118, 10900.

20 M. A. Palafox, M. Gill, N. J. Nunez, V. K. Rastogi, L. Mittal and R. Sharma, Int. J. Quantum Chem., 2005, 103, 394.

21 V. Hänninen and L. Halonen, J. Chem. Phys., 2007, 126, 064309.

22 I. V. Alabugin, M. Manoharan, M. Buck and R. J. Clark, THEOCHEM, 2007, 813, 21.

23 J. C. Jiang and C. E. Lin, THEOCHEM, 1997, 392, 181.

24 W. B. Tzeng, K. Narayanan, K. C. Shieh and C. C. Tung, THEOCHEM, 1998, 428, 231.

25 I. López-Tocón, R. G. della Valle, M. Becucci, E. Castellucci and J. C. Otero, Chem. Phys. Lett., 2000, 327, 45.

26 D. M. Upadhyay, M. K. Shukla and P. C. Mishra, THEOCHEM, 2000, 531, 249.

27 K. Pirowska, P. Kolekand, J. Goclon and J. Najbar, Chem. Phys. Lett., 2004, 387, 165.

28 G. Piani, M. Pasquini, I. López-Tocón, G. Pietraperzia, M. Becucci and E. Castellucci, Chem. Phys., 2006, 330, 138.

29 E. Drougas, J. G. Philis and A. M. Kosmas, THEOCHEM, 2006, 758, 17.

30 W. E. Sainclair and D. W. Pratt, J. Chem. Phys., 1996, 105, 7942.

31 L. Santos, E. Martinez, B. Ballesteros and J. Sanchez, Spectrochim. Acta, Part A, 2000, 56, 1905.

32 G. M. Roberts, C. A. Williams, J. D. Young, S. Ullrich, M. J. Paterson and V. G. Stavros, J. Am. Chem. Soc., 2012, 134, 12578.

33 H. V. Weyssenhoff and F. Kraus, J. Chem. Phys., 1971, 54, 2387.

34 R. Scheps, D. Florida and S. A. Rice, J. Chem. Phys., 1973, 61, 1730.

35 J. L. Knee and P. M. Johnson, J. Chem. Phys., 1984, 80, 13.

36 B. Kim, C. P. Schick and P. M. Weber, J. Chem. Phys., 1995, 103, 6903.

37 Y. Honda, M. Hada, M. Ehara and H. Nakatsuji, J. Chem. Phys., 2002, 117, 2045.

38 T. Ebata, C. Minejima and N. Mikami, J. Phys. Chem. A, 2002, 106, 11068.

39 X.-J. Hou, P. Quan, T. Höltzl, T. Veszprémi and M. T. Nguyen, J. Phys. Chem. A, 2005, 109, 10396.

40 F. Wang, S. P. Neville, R. Wang and G. A. Worth, J. Phys. Chem. A, 2013, 117, 7298.

41 G. A. King, T. A. A. Oliver and M. N. R. Ashfold, J. Chem. Phys., 2010, 132, 214307. 
42 R. Montero, A. P. Conde, V. Ovejas, R. Martínez, F. Castaño and A. Longarte, J. Chem. Phys., 2011, 135, 054308.

43 R. Spesyvtsev, O. M. Kirkby, M. Vacher and H. H. Fielding, Phys. Chem. Chem. Phys., 2012, 14, 9942.

44 R. Spesyvtsev, O. M. Kirkby and H. H. Fielding, Faraday Discuss., 2012, 157, 165.

45 J. O. F. Thompson, R. A. Livingstone and D. Townsend, J. Chem. Phys., 2013, 139, 034316.

46 I. N. Ragazos, M. A. Robb, F. Bernardi and M. Olivucci, Chem. Phys. Lett., 1992, 197, 217.

47 M. J. Bearpark, M. A. Robb and H. B. Schlegel, Chem. Phys. Lett., 1994, 223, 269.

48 F. Bernardi, M. A. Robb and M. Olivucci, Chem. Soc. Rev., 1996, 25, 321.

49 C. Gonzalez and H. B. Schlegel, J. Chem. Phys., 1989, 90, 2154.

50 C. Gonzalez and H. B. Schlegel, J. Phys. Chem., 1990, 94, 5523.

51 In theory, increasing the size of the active space can allow us to recover a part of the dynamical electronic correlation energy. However, in most cases, this strategy requires an active space too large to be computationally practical.

52 A. A. Granovsky, J. Chem. Phys., 2011, 134, 214113.

53 It is noteworthy that optimizations of excited state minima and conical intersections of similar compounds at the (MS-)MR-PT2 level of theory were performed in previous studies using numerical optimization procedures. ${ }^{110-113}$ The resulting structures and energetics were compared to CASSCF predictions. Overall, this studies suggested that, while it is known that the MS-MR-PT2//CASSCF standard procedure can yield dramatically different results than full CASSCF calculations, both in term of energetics and of mechanistic picture of the process under investigation, full MS-MR-PT2 calculations (both optimizations and potential energy scans) are not expected to significantly alter the conclusions obtained by MS-MR-PT2//CASSCF calculations. We mention, however, that in particularly tricky cases where the ordering of the states is very different from CASSCF to MS-MR-PT2, a full MS-MR-PT2 study can be the only solution to obtain reliable results. ${ }^{114}$ In addition, single reference methods such as $\mathrm{CC} 2^{115}$ and $\operatorname{ADC}(2)^{116}$ can be used to map excited state potential energy surfaces in regions free of strong multi-reference character. These methods are known to provide excitation energies and excited state minima of better quality than CASSCF. ${ }^{117}$.

54 L. Serrano-Andrés, M. Merchán and A. C. Borin, Chem.-Eur. J., 2006, 12, 6559.

55 L. Blancafort, J. Am. Chem. Soc., 2006, 128, 210.

56 M. Merchán and L. Serrano-Andrés, J. Am. Chem. Soc., 2003, 125, 8108.

57 L. Serrano-Andrés, M. Merchán and A. C. Borin, J. Am. Chem. Soc., 2008, 130, 2473.

58 H. Nakano, J. Chem. Phys., 1993, 99, 7983.

59 A. A. Granovsky, Firefly version 7.1.G, http://classic.chem. msu.su/gran/firefly/index.html.
60 E. Epifanovsky, I. Polyakov, B. Grigorenko, A. Nemukhin and A. I. Krylov, J. Chem. Phys., 2010, 132, 115104.

61 I. V. Polyakov, B. L. Grigorenko, E. M. Epifanovsky, A. I. Krylov and A. V. Nemukhin, J. Chem. Theory Comput., 2010, 6, 2377.

62 B. L. Grigorenko, A. V. Nemukhin, D. I. Morozov, I. V. Polyakov, K. B. Bravaya and A. I. Krylov, J. Chem. Theory Comput., 2011, 8, 1912.

63 B. L. Grigorenko, A. V. Nemukhin, I. V. Polyakov and A. I. Krylov, J. Phys. Chem. Lett., 2013, 4, 1743.

64 P. M. Kozlowski, T. Kamachi, M. Kumar, T. Nakayama and K. Yoshizawa, J. Phys. Chem. B, 2010, 114, 5928.

65 A. Udvarhelyi and T. Domratcheva, J. Photochem. Photobiol., A, 2011, 87, 554.

66 I. Ioffe, A. L. Dobryakov, A. A. Granovsky, N. P. Ernsting and J. L. P. Lustres, ChemPhysChem, 2011, 12, 1860.

67 A. V. Bochenkova and L. H. Andersen, Faraday Discuss., 2013, 163, 297.

68 K. Kornobis, N. Kumar, B. M. Wong, P. Lodowski, M. Jaworska, T. Andruni, K. Ruud and P. M. Kozlowski, J. Phys. Chem. A, 2011, 115, 1280.

69 S. Gozem, M. Huntress, I. Schapiro, R. Lindh, A. A. Granovsky, C. Angeli and M. Olivucci, J. Chem. Theory Comput., 2012, 8, 4069.

70 S. Gozem, F. Melaccio, R. Lindh, A. I. Krylov, A. A. Granovsky, C. Angeli and M. Olivucci, J. Chem. Theory Comput., 2013, 9, 4495.

71 F. Berndt, I. Ioffe, A. A. Granovsky, R. Mahrwald, S. Tannert, S. A. Kovalenko and N. P. Ernsting, J. Photochem. Photobiol., A, 2012, 234, 164.

72 A. L. Dobryakov, I. Ioffe, A. A. Granovsky, N. P. Ernsting and S. A. Kovalenko, J. Chem. Phys., 2012, 137, 244505.

73 A. L. Sobolewski, W. Domcke, C. Dedonder-Lardeux and C. Jouvet, Phys. Chem. Chem. Phys., 2002, 4, 1093.

74 M. N. R. Ashfold, G. A. King, D. Murdock, M. G. D. Nix, T. A. A. Oliver and A. G. Sage, Phys. Chem. Chem. Phys., 2010, 12, 1218.

75 V. Vallet, Z. Lan, S. Mahapatra, A. L. Sobolewski and W. Domcke, Faraday Discuss., 2004, 127, 283.

76 V. Vallet, Z. Lan, S. Mahapatra, A. L. Sobolewski and W. Domcke, J. Chem. Phys., 2005, 123, 144307.

77 Z. Lan, W. Domcke, V. Vallet, A. L. Sobolewski and S. Mahapatra, J. Chem. Phys., 2005, 122, 224315.

78 M. J. Frisch, G. W. Trucks, H. B. Schlegel, G. E. Scuseria, M. A. Robb, J. R. Cheeseman, J. A. Montgomery, Jr., T. Vreven, K. N. Kudin, J. C. Burant, J. M. Millam, S. S. Iyengar, J. Tomasi, V. Barone, B. Mennucci, M. Cossi, G. Scalmani, N. Rega, G. A. Petersson, H. Nakatsuji, M. Hada, M. Ehara, K. Toyota, R. Fukuda, J. Hasegawa, M. Ishida, T. Nakajima, Y. Honda, O. Kitao, H. Nakai, M. Klene, X. Li, J. E. Knox, H. P. Hratchian, J. B. Cross, V. Bakken, C. Adamo, J. Jaramillo, R. Gomperts, R. E. Stratmann, O. Yazyev, A. J. Austin, R. Cammi, C. Pomelli, J. W. Ochterski, P. Y. Ayala, K. Morokuma, G. A. Voth, P. Salvador, J. J. Dannenberg, V. G. Zakrzewski, S. Dapprich, A. D. Daniels, M. C. Strain, O. Farkas, 
D. K. Malick, A. D. Rabuck, K. Raghavachari, J. B. Foresman, J. V. Ortiz, Q. Cui, A. G. Baboul, S. Clifford, J. Cioslowski, B. B. Stefanov, G. Liu, A. Liashenko, P. Piskorz, I. Komaromi, R. L. Martin, D. J. Fox, T. Keith, M. A. Al-Laham, C. Y. Peng, A. Nanayakkara, M. Challacombe, P. M. W. Gill, B. Johnson, W. Chen, M. W. Wong, C. Gonzalez and J. A. Pople, Gaussian, Inc., Wallingford, CT, 2004.

79 M. W. Schmidt, K. K. Baldridge, J. A. Boatz, S. T. Elbert, M. S. Gordon, J. H. Jensen, S. Koseki, N. Matsunaga, K. A. Nguyen, S. Su, T. L. Windus, M. Dupuis and J. A. Montgomery, J. Comput. Chem., 1993, 14, 1347.

80 T. A. Stephenson, P. L. Radloff and S. A. Rice, J. Chem. Phys., 1984, 81, 1060.

81 T. A. Stephenson and S. A. Rice, J. Chem. Phys., 1984, 81, 1073.

82 I. J. Palmer, I. N. Ragazos, F. Bernardi, M. Olivucci and M. A. Robb, J. Am. Chem. Soc., 1993, 115, 673.

83 G. A. Worth, J. Photochem. Photobiol., A, 2007, 190, 190.

84 G. A. Worth, R. E. Carley and H. H. Fielding, Chem. Phys., 2007, 338, 220.

85 B. Lasorne, F. Silicia, M. J. Bearpark, M. A. Robb, G. A. Worth and L. Blancafort, J. Chem. Phys., 2008, 128, 124307.

86 B. Lasorne, M. J. Bearpark, M. A. Robb and G. A. Worth, J. Phys. Chem. A, 2008, 112, 13017.

87 D. S. N. Parker, R. S. Minns, T. J. Penfold, G. A. Worth and H. H. Fielding, Chem. Phys. Lett., 2009, 469, 43.

88 T. J. Penfold and G. A. Worth, J. Chem. Phys., 2009, 131, 064303.

89 R. S. Minns, D. S. N. Parker, T. J. Penfold, G. A. Worth and H. H. Fielding, Phys. Chem. Chem. Phys., 2010, 12, 15607.

90 T. J. Penfold, R. Spesyvtsev, O. M. Kirkby, R. S. Minns, D. S. N. Parker, H. H. Fielding and G. A. Worth, J. Chem. Phys., 2012, 137, 204310.

91 A. L. Sobolewski, C. Woywod and W. Domcke, J. Chem. Phys., 1992, 98, 5627.

92 O. P. J. Vieuxmaire, Z. Lan, A. L. Sobolewski and W. Domcke, J. Chem. Phys., 2008, 129, 224307.

93 The $1 \pi \pi^{*}$ and $2 \pi \pi^{*}$ states are bound and their energies increase significantly with the $\mathrm{N}-\mathrm{H}$ bond length. At large $\mathrm{N}-\mathrm{H}$ bond length, $1 \pi \pi^{*}$ and $2 \pi \pi^{*}$ state potential energy curves cross that of high lying dissociative states, including a number of states dominated by doubly excited configurations. This situation leads to considerable difficulty in keeping track of the states of interest and to convergence problems. Computing separately the $\mathrm{A}^{\prime}$ and $\mathrm{A}^{\prime \prime}$ states was found to greatly simplify the calculation since only crossings implying states of the same symmetry remained to be dealt with.

94 H. Köppel, W. Domcke and L. S. Cederbaum, Adv. Chem. Phys., 1984, 57, 59.

95 S. Matsika and D. R. Yarkony, J. Chem. Phys., 2002, 117, 6907.

96 S. Han and D. R. Yarkony, J. Chem. Phys., 2003, 119, 11561.

97 M. S. Schuurman and D. R. Yarkony, J. Phys. Chem. B, 2006, 110, 19031.

98 M. Barbatti, M. Vazdar, A. J. A. Aquino, M. Eckert-Maksić and H. Lischka, J. Chem. Phys., 2006, 125, 164323.

99 S. Perun, A. L. Sobolewski and W. Domcke, J. Am. Chem. Soc., 2005, 127, 6257.

100 H. Ward and J. Wishnok, J. Am. Chem. Soc., 1968, 90, 5353. 101 D. Bryce-Smith, A. Gilbert and D. A. Robinson, Angew. Chem., Int. Ed., 1971, 10, 745.

102 A. L. Sobolewski, J. Chem. Phys., 1990, 93, 6433.

103 A. Toniolo, A. L. Thompson and T. J. Martínez, Chem. Phys, 2004, 304, 133.

104 A. L. Thompson and T. J. Martínez, Faraday Discuss., 2011, 150, 293.

105 B. Lasorne, M. J. Bearpark, M. A. Robb and G. A. Worth, J. Phys. Chem. A, 2008, 112, 13017.

106 D. Mendive-Tapia, B. Lasorne, G. A. Worth, M. J. Bearpark and M. A. Robb, Phys. Chem. Chem. Phys., 2010, 12, 15725.

107 J. J. Serrano-Pérez, F. de Vleeschouwer, F. de Proft, D. Mendive-Tapia, M. J. Bearpark and M. A. Robb, J. Org. Chem., 2013, 78, 1874.

108 G. A. Worth, M. A. Robb and B. Lasorne, Mol. Phys., 2008, 106, 2077.

109 M. Barbatti, Wiley Interdiscip. Rev.: Comput. Mol. Sci., 2011, 1, 620 .

110 C. S. Page and M. Olivucci, J. Comput. Chem., 2003, 24, 298.

111 L. Serrano-Andrés, M. Merchán and R. Lindh, J. Chem. Phys., 2005, 122, 104107.

112 B. G. Levine, J. D. Coe and T. J. Martínez, J. Phys. Chem. B, 2008, 112, 405.

113 M. Huix-Rotllant, D. Siri and N. Ferré, Phys. Chem. Chem. Phys., 2013, 15, 19293.

114 I. N. Ioffe and A. A. Granovsky, J. Chem. Theory Comput., 2013, 9, 4973.

115 O. Christiansen, H. Koch and P. Jørgensen, Chem. Phys. Lett., 1995, 243, 409.

116 J. Schirmer, Phys. Rev. A: At., Mol., Opt. Phys., 1982, 26, 2395.

117 N. O. C. Winter, N. K. Graf, S. Leutwyler and C. Hättig, Phys. Chem. Chem. Phys., 2013, 15, 6623. 